COMECHINGONIA. REVISTA DE ARQUEOLOGÍA

Número 16 (2), segundo semestre 2012, pp. 139-153, Córdoba

ISSN 0326-7911

\title{
GUANACOS, SEXO Y EDAD. EL CASO DEL SITIO LAS VUELTAS 1 (TIERRA DEL FUEGO, ARGENTINA).
}

\section{GUANACOS, SEX AND AGE. THE CASE OF LAS VUELTAS 1 SITE (TIERRA DEL FUEGO, ARGENTINA)}

\author{
María Belén Colasurdo ${ }^{1}$, Fernando Santiago ${ }^{2}$ y Mónica Salemme ${ }^{3}$ \\ 1_-CESOR/ISHIR-CONICET. E.mail: belencolasurdo@gmail.com \\ ${ }^{2}$ CADIC-CONICET. E-mail: ersant2@gmail.com \\ ${ }^{3}$ CADIC-CONICET y UNTF. E-mail: monica.salemme@gmail.com
}

Presentado el: 24/08/2012 - Aceptado el: 07/11/2012

\begin{abstract}
Resumen
El sitio Las Vueltas 1 (Tierra del Fuego, Argentina) ha sido definido como sitio de matanza y procesamiento de guanacos; los diversos eventos de captura han dejado como registro un NISP total de 3404 huesos de guanaco (de los cuales 1751 provienen de la 3 ra ocupación), donde todas las partes esqueletarias están representadas diferencialmente. El objetivo de este trabajo es dar a conocer el sexo y la edad de los guanacos cazados durante la ocupación más reciente de este sitio considerando que el análisis de la estructura sexual y etaria de los conjuntos zooarqueológicos aporta valiosa información para identificar estrategias de caza, así como si las mismas se concentraron sobre algún grupo social, etario o sexual en particular. Para determinar el sexo y la edad de los guanacos de LV1 se tuvieron en cuenta análisis odontométrico y medidas osteométricas en pelvis, utilizando todos los fragmentos de maxilares, mandíbulas y de pelvis, recuperados en estratigrafía. La ocupación de la cual proviene el material está fechada en el Holoceno tardío.
\end{abstract}

Palabras claves: Guanaco, Estructura etaria y sexual, Estepa fueguina

\begin{abstract}
Las Vueltas 1 site (Tierra del Fuego, Argentina) has been characterized as a guanaco (Lama guanicoe) kill site, where guanacos were hunted and butchered; several hunting events left a total NISP of 3404 guanaco bones (being 1751 specimens from the 3rd. occupation); all the skeletal pieces are differentially represented. The main goal of this paper is to find out sex and age of guanacos coming from the latest occupation in the site, considering that the sexual and aging structure of $a$ zooarchaeological assemblage lights on the hunting strategies as well as if they were concentrated in a particular social, sexual or age group of animals, guanacos in this case. Odontometric analyses
\end{abstract}


and osteometric measures in pelvis were used to identify sex and age of the guanacos from LV1; all mandible, maxilla and pelvis fragments coming from stratigraphic position were employed. The studied material, coming from the third occupation in the site, dates from the Late Holocene.

Keywords: Guanaco, Sexual and aging structure, Fuegian steppe

\section{Introducción}

El sitio LV1 fue definido como sitio de matanza y procesamiento de guanacos, donde por lo menos tres momentos de ocupación han sido identificados. Para la tercera y más reciente ocupación se ha planteado la posibilidad de estar ante un evento único de matanza de guanacos (Santiago 2010), aunque también podría tratarse de unos pocos eventos de caza restringidos a un momento breve de ocupación. Por ello, determinar el sexo y la edad de los ejemplares presentes en la misma es de suma importancia, ya que permite interpretar sobre qué tipo de manadas se estaba predando en este momento (ca. 550 años AP, ver Santiago 2010), así como reconocer si hubo estacionalidad en la utilización del sitio. Es por esto que el análisis se realiza únicamente sobre los restos óseos asignados a la tercera ocupación, por ser los más numerosos así como por la posibilidad de que esta ocupación represente un mismo evento de caza, o unos pocos eventos separados por poco tiempo.

Para realizar dicho análisis se tomaron como marco metodológico los estudios realizados por Raedeke (1978), Puig y Monge (1983), Sarasqueta (2001), Kauffman (2008) y Kaufmann y L'Heureux (2009). Se tomaron medidas en diferentes partes esqueletales como pelvis, húmeros y radio- ulnas (las medidas en estos dos últimos elementos luego fueron descartadas por no ser diagnósticas, tal y como lo afirman Kaufmann y L'Heureux 2009) y una serie de medidas y observaciones en elementos dentarios: caninos, caniniformes, alvéolos, ramas mandibulares y molares. Según los autores consultados, algunas medidas arrojan datos más confiables que otras; por esto, para minimizar el margen de error en las interpretaciones, fueron relevadas la mayor cantidad de medidas posibles, aunque al momento del análisis y por diversas razones, algunas fueron descartadas.

Las conclusiones obtenidas, en todo caso preliminares ${ }^{1}$, acerca del tipo de manada sobre la que se estaba predando, permite plantear dos alternativas, ya que según los datos a tener en cuenta y cómo se crucen las variables, se llega a resultados distintos, sobre todo en lo que se refiere a la determinación de sexo. Dichas diferencias están sujetas además a los problemas metodológicos que existen a la hora de determinar dimorfismo sexual en guanacos. En lo que respecta al perfil etario éste fue definido con más precisión, ya que los datos obtenidos son más claros y los métodos utilizados son más efectivos.

\section{Descripción del sitio Las Vueltas 1}

El sitio Las Vueltas 1 (en adelante LV1) se encuentra ubicado a $4 \mathrm{~km}$ en línea recta de la costa atlántica fueguina; fue descubierto durante prospecciones sistemáticas (Santiago et al. 2007), en las que se observaron grandes cantidades de huesos de guanaco y materiales líticos en superficie. Los materiales arqueológicos se encuentran en una duna limo-arcillosa donde la acción eólica generó una cubeta de deflación de 35 a $40 \mathrm{~cm}$ de profundidad, que abarca una superficie de unos 600 m2 (Santiago y Salemme 2009). En 2005 esta hoyada estaba completamente despojada de cobertura vegetal, en tanto en la actualidad,se encuentra totalmente re-vegetada. 


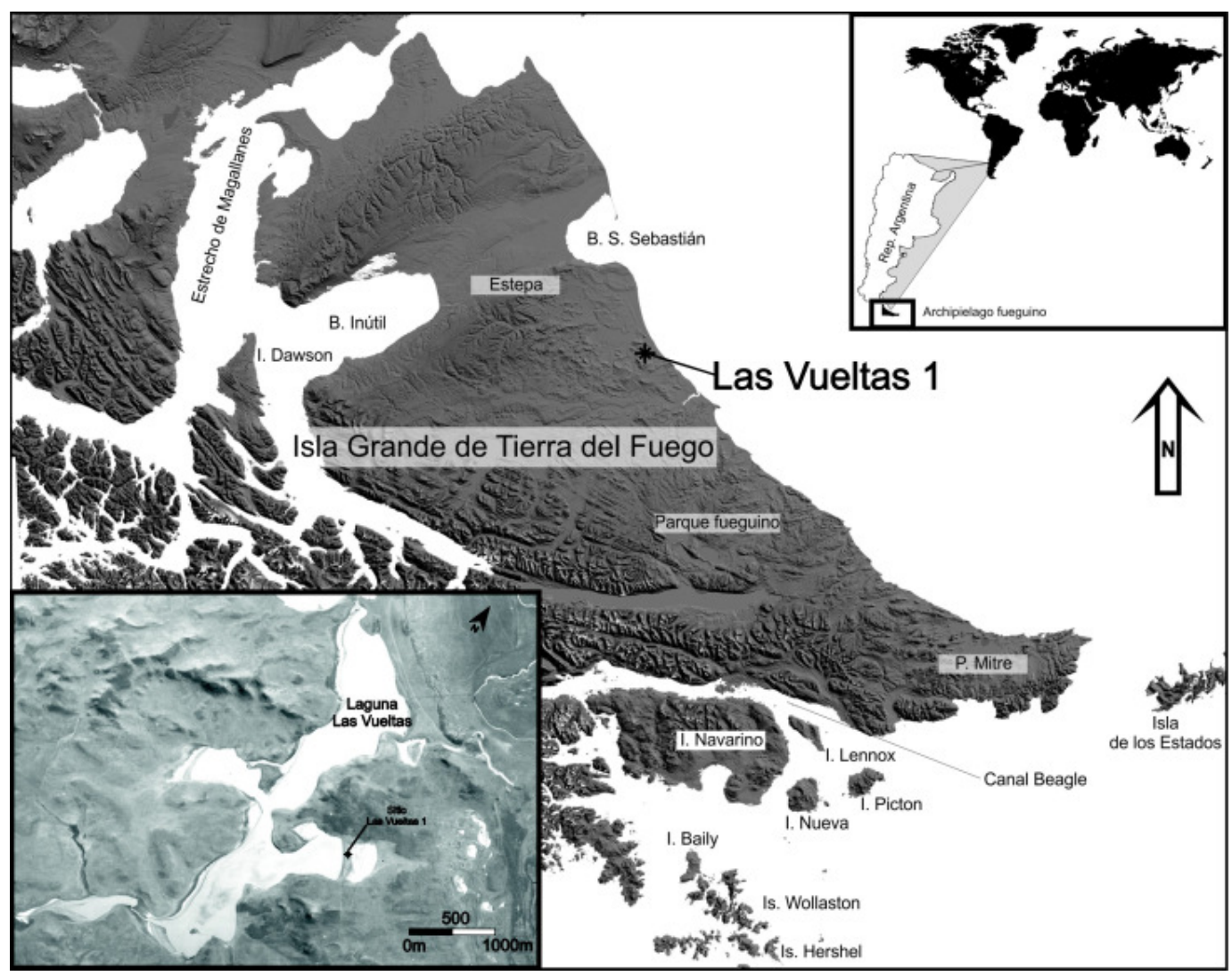

Figura 1. Ubicación del sitio Las Vueltas 1, en la Isla Grande de Tierra del Fuego

El sitio está emplazado en un "istmo" de tierra entre dos lagunas, rodeadas por elevaciones de origen terciario lo cual da lugar a un paisaje particular, similar a un anfiteatro que se abre hacia el oeste. En la actualidad, los campos son utilizados para pastoreo de ganado ovino, y es frecuente observar los rebaños pastando y bebiendo en este sector, donde además se ha instalado un "brete" para encerrar al ganado (Figura 1).

El guanaco es la especie más significativa en esta ocupación, con 1750 especímenes identificados (Tabla 1), en tanto el NISP de tuco-tuco (Ctenomys magellanicus) alcanza 1242 restos; la categoría siguiente en cantidad está representada por fragmentos indeterminados de mamíferos terrestres con 1096 especímenes, los cricétidos suman 590 especímenes mientras que 511 restos son huesos indeterminados carbonizados; en mucha menor proporción se registraron huesos de ave $(\mathrm{N}=65)$ , cánido $(\mathrm{N}=21)$, lobo marino $(\mathrm{N}=5)$, peces $(\mathrm{N}=2)$ y cetáceo $(\mathrm{N}=1)$. Además se recuperaron 188 valvas de Mytilus edulis, 47 de Nacella sp. y 2 de Aulacomya atra (Santiago 2010).

El número mínimo de individuos de guanaco identificados para la $3^{\text {ra }}$ ocupación es de 15 animales, calculado en base a los fragmentos de diáfisis de tibia. En la cama de huesos hay representado un amplio espectro de partes esqueletarias de Lama guanicoe. Están presentes cráneos, vértebras, huesos de la pelvis y de ambas extremidades. Los huesos del esqueleto apendicular (NISP=481- MNE 352) son más numerosos que los del axial (NISP=375 MNE=227). La unidad anatómica de mayor frecuencia está representada por las costillas con un MNE de 66 (Tabla 2). 


\begin{tabular}{l|l|c|c|c|} 
Nombre vulgar & \multicolumn{1}{|c|}{ Taxón } & NISP & \%NISP & MNI \\
\hline Tuco-Tuco & Ctenomys magellanicus & 1242 & 23,5 & 68 \\
Guanaco & Lama guanicoe & 1750 & 33,1 & 15 \\
Roedor & Cricetidae & 590 & 11,2 & 48 \\
Zorro & Canidae & 21 & 0,4 & 1 \\
Ave & Ave & 65 & 1,2 & - \\
Mamífero terrestre & Mammalia & 1096 & 20,7 & - \\
Lobo Marino & Pinnipedia & 5 & 0,1 & 1 \\
Delfin & Cetacea & 1 & 0,0 & 1 \\
Peces & Osteichthyes & 2 & 0,0 & 1 \\
Hueso quemado & Mammalia INDET & 511 & 9,7 & - \\
\hline \multicolumn{2}{l|}{ TOTAL } & $\mathbf{5 2 8 3}$ & $\mathbf{1 0 0}$ & - \\
\hline
\end{tabular}

Tabla 1: Representación taxonómica NISP, \%NISP y MNI de los taxones identificados en la 3era ocupación de LV1.

Una breve síntesis de cómo se encontraban depositados los huesos de guanaco y cómo se asociaban entre sí se menciona a continuación. Una caracterización más completa del contexto, tanto composición de partes esqueletarias, tafonomía, marcas antrópicas e integridad del conjunto se detalla en Santiago (2010).Los cráneos encontrados se presentan fracturados y los occipitales -en 4 ocasiones- yacían anatómicamente asociados a su correspondientes atlas y axis. En cuanto al resto, las $1^{\text {eras }}$ y $2^{\text {das }}$ vértebras, o remontaban anatómicamente con la $3^{\text {ra }}$ cervical o se encontraban muy próximas unas de otras, indicando una desarticulación incipiente.

Las restantes partes de la columna vertebral se encontraban asociadas anatómicamente de a 4 ó 5 vértebras, destacándose en las vértebras lumbares la supresión -por medio de golpes- de las apófisis transversas.

En todas las mandíbulas se identificaron golpes en su parte central, evidenciados por puntos de impacto. Se encontraron muy pocos dientes y molares aislados; la mayor parte están en sus correspondientes alvéolos y los que se encuentran sueltos generalmente están fracturados, hecho que se puede asociar con los puntos de impacto antes mencionados, y permite interpretar que los dientes se desprendieron de su lugar por el o los golpes recibidos. La presencia de los dientes en sus respectivas mandíbulas es un buen indicador de que no hubo muchos cambios postdepositacionales en la muestra, por ejemplo por pisoteo.

Con respecto a las costillas, se pudieron identificar todas desde la $1^{\text {era }}$ hasta la $12^{\text {ava }}$, y de ambos lados del esqueleto, siendo la más representada la $10^{\mathrm{ma}}$; muchas de ellas se presentan enteras y con marcas de corte, tanto en sus porciones articulares como en su parte medial.

Todos los huesos largos de guanaco se encontraron fracturados; las fracturas de los huesos largos presentan un patrón claro de acción humana, eligiendo a los metapodios y 1eras falanges, para fracturarlos longitudinalmente y a húmero, radiocúbito, fémur y tibia para hacerlo transversalmente; en todos los huesos -en diferentes proporciones- se observan marcas de corte, puntos de impacto, negativos de lascado, quemado, carbonizado y surco perimetral (Santiago 2010). 
El sitio se ha caracterizado como de "trampa activa" (sensu Marean 1997), un lugar apropiado para arriar, concentrar y matar guanacos. Luego de la matanza el mismo lugar se transformaba en un lugar de procesamiento y consumo de los animales cazados (Santiago 2010; Santiago y Salemme 2009, 2010).

Por tratarse del conjunto cuantitativamente más numeroso y complejo, este trabajo circunscribe su objetivo en los resultados que surgen del análisis zooarqueológico de la especie mejor representada en el tercer evento de ocupación, el guanaco (Lama guanicoe), y

\begin{tabular}{|c|c|c|c|c|c|c|c|c|c|c|c|c|c|c|c|c|c|}
\hline \multirow{2}{*}{$\begin{array}{l}\text { Unidad } \\
\text { anatómica }\end{array}$} & \multicolumn{4}{|c|}{ Inmaduro } & \multicolumn{4}{|c|}{ Maduro } & \multicolumn{4}{|c|}{ Indeterminado } & \multirow{2}{*}{$\begin{array}{l}\text { NISP } \\
\text { Total }\end{array}$} & \multirow{2}{*}{ MNI } & \multirow{2}{*}{$|\mathrm{MNE}|$} & \multirow{2}{*}{ MAU } & \multirow{2}{*}{$\begin{array}{c}\% \\
\text { MAU }\end{array}$} \\
\hline & $\mathrm{Iz}$ & $\overline{\text { Dr }}$ & $\overline{\mathrm{Ax}}$ & Indt & $\mathrm{Iz}$ & $\overline{\mathrm{Dr}}$ & $\mathrm{Ax}$ & Indt & \begin{tabular}{|l}
$\mathrm{Iz}$ \\
\end{tabular} & $\overline{\text { Dr }}$ & $\overline{\mathrm{Ax}}$ & Indt & & & & & \\
\hline$\overline{\text { Cráneo }}$ & - & - & - & - & 3 & 5 & 15 & - & - & 1 & 4 & 4 & 32 & 9 & 9 & 9 & 72 \\
\hline Incisivo & - & - & - & - & - & - & - & - & - & - & - & 6 & 6 & - & - & - & - \\
\hline Canino & - & - & - & - & - & - & - & 1 & - & - & - & - & 1 & 1 & 1 & - & - \\
\hline Molar & - & - & - & - & - & - & - & 13 & - & - & - & - & 13 & 2 & 6 & & 0 \\
\hline Maxilar & 1 & - & - & - & 7 & 2 & - & - & - & - & - & - & 10 & 8 & 10 & 5 & 40 \\
\hline Hemimandíbula & - & - & - & - & 12 & 8 & 4 & - & - & 1 & - & 3 & 28 & 11 & 20 & 10 & 80 \\
\hline Atlas & - & - & - & - & - & - & 8 & - & - & - & - & - & 8 & 8 & 8 & 8 & 64 \\
\hline Axis & - & - & 3 & - & - & - & 4 & - & - & - & - & - & 7 & 7 & 7 & 7 & 56 \\
\hline Cervical & - & - & 11 & - & - & - & 16 & - & - & - & 1 & - & 28 & 6 & 27 & 5,4 & 43,2 \\
\hline Toráxica & - & - & 21 & - & - & - & 27 & - & - & - & - & - & 48 & 4 & 39 & 3,3 & 26 \\
\hline Lumbar & - & - & 3 & - & - & - & 36 & - & - & - & - & - & 39 & 6 & 24 & 3,4 & 27,4 \\
\hline Sacra & - & - & - & - & - & - & 5 & - & - & - & - & - & 5 & 5 & 5 & 5 & 40 \\
\hline Caudal & - & - & - & - & - & - & - & - & - & - & - & - & - & - & - & - & - \\
\hline Vértebra Indet. & - & - & 34 & - & - & - & 9 & - & - & - & 12 & & 55 & - & - & - & - \\
\hline Costilla & 1 & 1 & - & 2 & 35 & 37 & - & 2 & 1 & 5 & - & 58 & 142 & 8 & 67 & 2,8 & 22,3 \\
\hline Esternebra & - & - & 3 & - & - & - & 1 & - & - & - & 1 & - & 5 & 4 & 4 & 4,0 & 32 \\
\hline Escápula & - & - & - & - & 4 & 14 & - & - & - & - & - & - & 18 & 10 & 12 & 6 & 48 \\
\hline Humer & 1 & 3 & - & - & 10 & 8 & - & - & - & - & - & - & 22 & 11 & 21 & 10,5 & 84 \\
\hline Húmero Ds. & - & - & - & - & 11 & 5 & - & - & - & - & - & - & 16 & 10 & 16 & 8 & 64 \\
\hline Húmero Diaf. & - & - & - & - & 2 & 4 & - & - & 8 & 6 & - & 1 & 21 & 13 & 23 & 11,5 & 92 \\
\hline Húmero comp. & - & - & - & - & - & - & - & - & - & - & - & - & - & - & - & - & - \\
\hline Radiocub. Px. & - & - & - & - & 3 & 4 & - & - & - & - & - & - & 7 & 4 & 7 & 3,5 & 28 \\
\hline Radiocub. Ds. & 1 & - & - & - & 4 & 2 & - & - & - & - & - & - & 7 & 5 & 7 & 3,5 & 28 \\
\hline Radiocub. Diaf. & - & - & - & - & 12 & 1 & - & - & 11 & 10 & - & 2 & 36 & 10 & 19 & 9,5 & 76 \\
\hline Radioc & - & - & - & - & - & - & - & - & - & - & - & - & - & - & - & - & - \\
\hline Pisciforme & - & - & - & - & 3 & 2 & - & - & - & - & - & - & 5 & 3 & 5 & 2,5 & 20 \\
\hline Escafoides & - & - & - & - & - & 1 & - & - & - & - & - & - & 1 & 1 & 1 & 0,5 & 4 \\
\hline Cuneiform & - & - & - & - & 3 & 4 & - & 2 & - & - & - & - & 9 & 6 & 9 & 4,5 & 36 \\
\hline Lunar & - & - & - & - & 2 & 1 & - & - & - & - & - & - & 3 & 2 & 3 & 1,5 & 12 \\
\hline Magnur & - & - & - & - & 1 & 2 & - & - & - & - & - & - & 3 & 2 & 3 & 1,5 & 12 \\
\hline Trapezoide & - & - & - & - & 1 & 4 & - & - & - & - & - & - & 5 & 4 & 5 & 2,5 & 20 \\
\hline Unciforme & - & - & - & - & 3 & 4 & - & - & - & - & - & - & 7 & 4 & 7 & 3,5 & 28 \\
\hline Metacarp. P & - & - & - & - & 4 & 8 & - & - & - & - & - & - & 12 & 8 & 8 & 4 & 32 \\
\hline Metacarp. Diaf. & - & - & - & - & 2 & - & - & - & - & - & - & - & 2 & 2 & 2 & 1 & 8 \\
\hline Hemipelvis & 1 & - & - & - & 6 & 5 & - & 1 & 1 & - & - & - & 14 & 5 & 6 & 3 & 24 \\
\hline Fémur Px. & - & - & - & - & 4 & 4 & - & - & - & - & - & - & 8 & 4 & 8 & 4 & 32 \\
\hline Fémur Ds. & - & - & - & - & 2 & 5 & - & - & - & - & - & - & 7 & 5 & 7 & 3,5 & 28 \\
\hline Fémur Diaf. & - & - & - & - & - & 1 & - & 2 & 8 & 10 & - & 7 & 28 & 6 & 17 & 8,5 & 68 \\
\hline
\end{tabular}

Tabla 2. Partes esqueletarias de Lama guanicoe: NISP, MNE, MAU, \%MAU y clases de edad de la 3era ocupación de LV1. 


\begin{tabular}{|c|c|c|c|c|c|c|c|c|c|c|c|c|c|c|c|c|c|}
\hline Tibia Px. & 4 & 8 & - & - & 7 & 4 & - & - & - & - & - & - & 23 & 12 & 23 & 11,5 & 92 \\
\hline Tibia Ds. & 1 & 3 & - & - & 2 & 3 & - & - & - & - & - & - & 9 & 6 & 9 & 4,5 & 36 \\
\hline Tibia Diaf. & - & - & - & - & - & 2 & - & 1 & 23 & 8 & - & 2 & 36 & 15 & 25 & 12,5 & 100 \\
\hline Astrágalo & - & - & - & - & 4 & - & - & - & - & - & - & - & 4 & 4 & 4 & 2 & 16 \\
\hline Calcáneo & 1 & 1 & - & - & 4 & 1 & - & - & - & - & - & - & 7 & 5 & 7 & 3,5 & 28 \\
\hline Endocuneiforme & - & - & - & - & 3 & 3 & - & - & - & - & - & - & 6 & 3 & 6 & 3 & 24 \\
\hline Navicular & - & - & - & - & 6 & 1 & - & - & - & - & - & - & 7 & 6 & 7 & 3,5 & 28 \\
\hline Ectocuneiforme & - & - & - & - & 3 & 1 & - & - & - & - & - & - & 4 & 3 & 4 & 2 & 16 \\
\hline Cuboide & - & - & - & - & 3 & 4 & - & - & - & - & - & - & 7 & 4 & 7 & 3,5 & 28 \\
\hline Metatar. Px. & - & - & - & - & 6 & 5 & - & - & - & - & - & - & 11 & 6 & 10 & 5 & 40 \\
\hline Metatar. Diaf. & - & - & - & - & - & 2 & - & - & - & - & - & - & 2 & 1 & 2 & 1 & 8 \\
\hline Metapod. Ds. & - & - & - & 3 & - & - & - & 9 & - & - & - & 2 & 14 & 3 & 13 & 3,25 & 26 \\
\hline Metapod. Diaf. & - & - & - & - & - & - & - & 3 & - & - & - & 46 & 49 & - & - & - & - \\
\hline $1^{\mathrm{e}}$ Falange & - & - & - & 1 & - & - & - & 28 & - & - & - & 2 & 31 & 4 & 23 & 2,9 & 23 \\
\hline $2^{\text {da }}$ Falange & - & - & - & 1 & - & - & - & 16 & - & - & - & - & 17 & 3 & 16 & 2 & 16 \\
\hline $3^{\mathrm{er}}$ Falange & - & - & - & - & - & - & - & 8 & - & - & - & 1 & 9 & 1 & 9 & 1,1 & 9 \\
\hline Diáfisis Indet. & - & - & - & - & - & - & - & 2 & - & - & - & 553 & 553 & - & - & - & - \\
\hline Hueso esponjoso & - & - & - & - & - & - & - & - & - & - & - & 51 & 51 & - & - & - & - \\
\hline Hueso plano & - & - & - & - & - & - & - & - & - & - & - & 68 & 68 & - & - & - & - \\
\hline Sesamoideo & - & - & - & - & - & - & - & - & - & - & - & 13 & 13 & - & - & - & - \\
\hline Lasca ósea & - & - & - & - & - & - & - & - & - & - & - & 171 & 171 & - & - & - & - \\
\hline \multirow{2}{*}{ TOTAL } & 11 & 16 & 75 & 7 & 169 & 152 & 110 & 74 & 52 & 40 & 14 & 980 & \multirow{2}{*}{1698} & \multirow{2}{*}{15} & \multirow{2}{*}{562} & \multirow{2}{*}{-} & \multirow{2}{*}{-} \\
\hline & \multicolumn{3}{|c|}{109} & & & \multicolumn{2}{|c|}{505} & & \multicolumn{4}{|c|}{1086} & & & & & \\
\hline
\end{tabular}

Tabla 2 (continuación). Partes esqueletarias de Lama guanicoe: NISP, MNE, MAU, \%MAU y clases de edad de la $3^{\text {era }}$ ocupación de LV1.

en particular de fragmentos de mandíbulas, maxilares y pelvis con el fin de determinar el sexo y la edad de los animales cazados en este contexto. En la Tabla 2 se presentan los datos de todas las unidades anatómicas de guanaco, destacando en gris las partes analizadas en el presente trabajo. Se ha analizado el porcentaje total de restos encontrados de pelvis, mandíbulas y maxilares. Es de destacar que las mandíbulas tienen valores altos de \%MAU en tanto los maxilares valores medios y las pelvis valores bajos.

\section{Variables de análisis}

Tal como se mencionó, para determinar el sexo de los guanacos presentes en la muestra de LV1 se tuvieron en cuenta, por un lado el análisis odontométrico y por el otro, las medidas osteométricas en pelvis.

El análisis odontométrico consistió en medir los caninos y los incisivos caniniformes, siguiendo las medidas propuestas por Kaufmann (2008, 2009: 113- Figura 33): Longitud total del canino (LtC), Longitud de la raíz del canino (LrC), Diámetro Mesiodistal del canino (DmdC), Diámetro Bucolingual del canino (DblC) (Tabla 3). Es importante destacar que tanto Kaufmann (2008) como Raedecke (1978) señalan que las medidas de los caninos superiores, así como de los incisivos caniniformes son menos confiables que los de las mandíbulas, porque hay una superposición de edad y sexo hasta los tres años. No obstante, para este análisis se incluyeron los datos para los caniniformes en aquellos casos que no se encuentran presentes los caninos, para poder así manejar una muestra de datos más numerosa y significativa. 
Siguiendo los rangos de valores para cada sexo, también según Kaufmann (2008, ver Tabla 3) fueron tomadas las medidas correspondientes a los caninos. En algunos casos los caninos se encontraban dentro de su alvéolo, y no pudieron ser extraídos sin romper sus respectivas mandíbulas, por lo tanto, fueron consideradas sólo las medidas que podían ser tomadas sin extraerlos.

\begin{tabular}{l|c|c|}
\multirow{2}{*}{\multicolumn{1}{c|}{ Variables }} & \multicolumn{2}{|c|}{ Rango de valores para } \\
\cline { 2 - 3 } & Hembra & Macho \\
\hline LtC $(\mathbf{m m})$ & $14,62-22,78$ & $23,33-34,73$ \\
LrC (mm) & $11,11-18,23$ & $22,12-33,12$ \\
DmdC (mm) & $4,11-6,87$ & $7,04-11,96$ \\
DldC (mm) & $2,58-4,02$ & $4,07-6,19$ \\
\hline
\end{tabular}

Tabla 3. Rango de valores odontométricos característicos de machos y hembras (tomado de Kaufmann 2008)

En cuanto a otras medidas osteométricas para la determinación del sexo, se tomaron las medidas que más claramente presentan diferencias significativas para la discriminación sexual, según Kaufmann y L'Heureux (2009). Estos autores determinaron que las principales diferencias osteométricas se relacionan con variaciones del tamaño y la forma de la pelvis completa y del acetábulo, para lo cual se toman las siguientes medidas: Altura de la espina isquiática (HIS) y Altura de la pared medial del acetábulo (HAM) (Ver Figura 1 en Kaufmann y L'Heureux 2009:183 y Tabla 4).

\begin{tabular}{c|c|c|}
\multirow{2}{*}{ Medida } & \multicolumn{2}{|c|}{ Valores medios } \\
\cline { 2 - 3 } & Macho & Hembra \\
\hline HIS $(\mathrm{mm})$ & 41,77 & 35,5 \\
HAM $(\mathrm{mm})$ & 19 & 16,55 \\
\hline
\end{tabular}

Tabla 4. Valores de las medidas medias para las variables HIS y HAM en pelvis según el sexo (Kaufmann y L'Heureux 2009).

En lo que respecta a la determinación de la edad, se tuvieron en cuenta una serie de variables relacionadas con la dentadura, tanto en los molares como en los incisivos.

Utilizando el método desarrollado por Puig y Monge (1983): Largo de la serie mandibular (LSM) y desgaste de los incisivos y molares. También se aplicaron estas medidas a los maxilares; pero al carecer de un método determinado como el utilizado con las mandíbulas, fue necesario cruzar varios datos tomados de diferentes autores.

Por un lado, siguiendo un trabajo sobre cría de guanacos actuales (Sarasqueta 2001) se realizó un rango de edad relacionado con la aparición o no de los caninos. Este autor señala que los caninos superiores no cortan hasta pasados los 26 meses y que entre los 36 y 38 meses ya están totalmente erupcionados. Considerando estos datos se estableció un rango de edad: 1) los maxilares con desarrollo completo de caninos fueron asignados a ejemplares de más de 36 meses, 2) los maxilares con caninos apenas erupcionados fueron 
determinados como ejemplares de entre 28 y 35 meses y 3) los maxilares con caninos que no cortaron indican un rango de menos de 26 meses.

También se tuvieron en cuenta los datos proporcionados por Raedeke (1978) sobre cómo se conforma la serie molar del maxilar:

1 canino y 1 incisivo caniniforme

PM 2 como deciduo que no es reemplazado por otro permanente

PM 3 Bicúspico como deciduo, remplazado por uno permanente de tipo unicúspico, que se pierde al llegar a la adultez.

PM 4 Bicúspico siempre, -deciduo y permanente

Molares: iguales a los de la mandíbula, excepto el M3 que no tiene el tubérculo adicional.

Dicho esto, cabe señalar que se utilizó el LSM y el desgaste de los molares (Puig y Monge 1983), considerando que el desarrollo del desgaste es similar en mandíbulas y maxilares (sensu Raedeke 1978). Sin embargo, otros autores observaron que maxilares y mandíbulas presentan desgastes totalmente distintos (Pagano y Aguerre 2001).

\section{Resultados}

Aunque se tomaron todas las variables posibles para la determinación de sexo (medidas de caninos, caniniformes, alvéolos, pelvis, húmeros, radiulnas) y edad (largo de la serie mandibular, estado de erupción dental, molar e incisivos), algunas de estas medidas presentan mayores problemas a la hora de definir indiscutiblemente la edad y el sexo y fueron descartadas. Las medidas tomadas en húmeros y radioulnas, fueron descartadas porque según lo expresado por Kaufmann y L'Heureux (2009) no muestran a nivel estadístico varianzas considerables entre sexos, por lo tanto no son determinantes claros para la diferenciación.

Como fue señalado anteriormente, aunque se tuvieron en cuenta para el análisis las medidas tomadas en los caninos superiores e incisivos caniniformes, según lo expresado por Kaufmann (2008) y Raedeke (1978) no son del todo confiables como las de las mandíbulas, porque hay una superposición de edad y sexo hasta los tres años. Es por ello que nos acotaremos a los datos que según la bibliografía son los más confiables para la determinación del sexo (Kaufmann 2008; Kaufmann y L'Heureux 2009).

\section{Determinación del sexo de los guanacos}

Las medidas obtenidas en caninos e incisivos caniniformes se presentan en la tabla 5. En dicha tabla se presenta la determinación sexual de cada uno de los especímenes. Cuando un especimen cuenta con más de una variable y dan resultados dudosos, o divergentes, se optó por tomar la variable que arrojó mejores resultados para la discriminación sexual en el modelo de referencia. Es decir siempre se prefirió utilizar el largo de la raíz ( $\mathrm{LrC}$ ) o el largo total ( $\mathrm{LtC})$ antes que el diámetro mesiodistal (DmdC) y el diámetro bucolingual (DblC).

Estos datos permiten entonces, siguiendo el rango citado por Kaufmann (2008), identificar en los maxilares 3 hembras (60\%) y 2 machos (40\%). Si se tienen en cuenta solo los caninos de las mandíbulas, por ser el dato más confiable para la diferenciación sexual, se identifican 4 machos (100\%). 


\begin{tabular}{|c|c|c|c|c|c|c|c|c|c|c|c|}
\hline \multirow{2}{*}{ Sigla } & \multirow{2}{*}{ Especimen } & \multirow{2}{*}{ Elemento } & \multicolumn{4}{|c|}{ Izquierdos } & \multicolumn{4}{|c|}{ Derechos } & \multirow[b]{2}{*}{ Sexo } \\
\hline & & & LtC & LrC & DmdC & $\overline{\text { DblC }}$ & LtC & LrC & DmdC & DblC & \\
\hline F41 143 & Canino & \multirow{4}{*}{ Mandíbula } & 28,9 & 13,2 & 6 & 4 & 0 & 0 & 0 & - & Macho \\
\hline F41 621 & Canino & & 28 & 18,2 & 4,5 & 2,6 & 25,1 & 14,7 & 4,8 & 3,1 & Macho \\
\hline F41 497 & Canino & & 27,9 & 25,8 & 10,9 & 6 & 28,5 & 26,9 & 10 & 6 & Macho \\
\hline E41 144 & Canino & & 0 & 0 & 9,5 & 5,1 & 0 & 0 & 9 & 5,5 & Macho \\
\hline$\overline{\text { F41sd }}$ & Canino & \multirow{5}{*}{ Maxilar } & 29,1 & 18,2 & $\overline{5,8}$ & 3,5 & 0 & 0 & 0 & - & Macho \\
\hline F41 588 & Canino & & 0 & - & 9 & 5,3 & 25,2 & 18,3 & 9,8 & 5,8 & Macho \\
\hline F41 526 & Canino & & 17 & 13,8 & 4 & 2,4 & 19 & 15 & 5 & 3 & Hembra \\
\hline F41 24 & Canino & & 0 & 28,9 & 5,1 & 3 & 0 & 0 & 5,5 & 2,6 & Hembra \\
\hline F41 590 & Caniniforme & & 15 & 11,1 & 4,2 & 3,2 & 0 & 0 & 0 & - & Hembra \\
\hline
\end{tabular}

Tabla 5. Variables para determinación del sexo según las medidas de caninos y caniniformes

En lo que se refiere al análisis de las pelvis de esta muestra (Tabla 6), los parámetros utilizados son más claros a la hora de los resultados, que por cierto revisten diferencias respecto a aquellos logrados a través de las piezas dentarias.

No se pudieron utilizar los métodos propuestos por Raedecke (1978) de observar la sínfisis púbica y la tuberosidad suspensoria del isquium para diferenciar el sexo, ya que las pelvis se encontraron fragmentadas. En la tabla 6 se puede observar que en todos los casos el acetábulo del fragmento de pelvis medido se encontraba fusionado.

Siguiendo los valores medios para cada medida (comparar con Tabla 4) se identificó que de un total de 9 especímenes medidos, 7 se acercan a la media determinada para machos $(78 \%)$ y 2 a la media determinada para hembras (22\%). Teniendo en cuenta la lateralidad de los mismos se podría identificar un MNI de 4 machos (80\%) y 1 hembra (20\%). Hay que tener en cuenta que las medidas de referencia fueron obtenidas en individuos mayores a 30 meses, pero si se miden individuos juveniles podría existir la posibilidad de que se determine como hembra a un macho juvenil (Kaufmann com. pers. 2012), aunque en este caso todos los acetábulos se encontraban fusionados como se observa en la tabla 6 .

\begin{tabular}{c|c|c|c|c|c|} 
Especimen & HIS (mm) & HAM (mm) & Sexo & Lateralidad & Fusión \\
\hline F41 551 & 41 & 20,5 & Macho & Dr & fusionado \\
F41 674 & 39,6 & - & Macho & Iz & fusionado \\
F41 261 & 41 & 18 & Macho & Dr & fusionado \\
F41 824 & 40,4 & 18,5 & Macho & Dr & fusionado \\
E42 319 & 40,9 & 18 & Macho & Iz & fusionado \\
F41 809 & 41,9 & 18,8 & Macho & Dr & fusionado \\
F41 429 & 41,3 & 19,3 & Macho & Iz & fusionado \\
E42 178 & 38 & - & Hembra & Iz & fusionado \\
F41 406 & 36 & 16 & Hembra & Dr & fusionado \\
\hline
\end{tabular}

Tabla 6. Identificación sexual según medidas en pelvis procedentes de la tercera ocupación de LV1. 


\section{Determinación de edad}

Los resultados se obtuvieron habiendo medido el largo de la serie mandibular (LSM), desgaste de los molares, desgaste de los incisivos y la edad estimada y se presentan en la tabla 7. El agrupamiento de los ejemplares logrado para los diversos rangos etarios se ilustra en la figura 2A, siguiendo la propuesta de Puig y Monje (1983) y en la figura 2B se puede observar las categorías de edad divididas en solo tres categorías según Stiner (1990), utilizando el método y el programa propuesto por Weaver et al. (2011).

Dado el cruce de las variables tomadas, en el conjunto de la tercera ocupación del sitio no se observan individuos recién nacidos, ni tampoco menores a 6 meses. Se identifican ejemplares en las categorías Crías y Juveniles (sensu Puig y Monje 1983), distribuidos de la siguiente forma: Cría 3: un ejemplar de 6 meses, Cría 4: un ejemplar de 7 a 9 meses, Cría 5: un ejemplar de 10 a 12 meses. Con respecto a la categoría Juveniles, se identificaron en Juvenil 1: 3 ejemplares entre 16 y 20 meses y en Juvenil 2: 2 ejemplares entre 21 y 26 meses. Los sub-adultos están representados por 2 ejemplares de 27 a 36 meses y en la categoría Adultos solamente están representados la de Adulto 1: con 2 ejemplares de 37 a 49 meses y la de Adulto 2: 2 ejemplares de 50 a 85 meses (ver Tabla 7 y Figura 2)
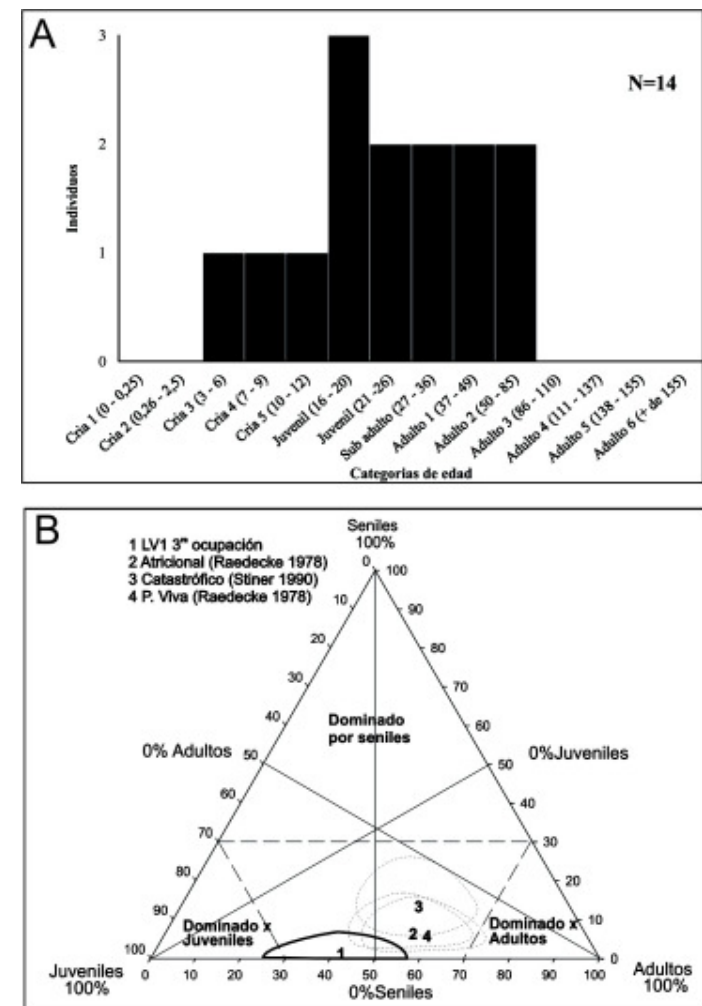

Figura 2. A - NMI reconocidos según categorías de edad en meses. B - Gráfico tripolar donde se agruparon en tres rangos de edad los animales identificados: juveniles (0-24 meses), adultos reproductivos (24-84 meses) y seniles (más de 84 meses). Se compara el perfil de LV1 con perfiles atricional, catastrófico y de población viva. 


\begin{tabular}{|c|c|c|c|c|c|c|c|}
\hline Sigla & Elemento & Sector & Lateralidad & $\begin{array}{l}\text { LSM } \\
(\mathrm{mm})\end{array}$ & $\begin{array}{l}\text { Molares } \\
\text { (meses) }\end{array}$ & $\mid \begin{array}{r}\text { Incisivos } \\
\text { (meses) }\end{array}$ & $\begin{array}{c}\text { Edad } \\
\text { (meses) }\end{array}$ \\
\hline F41497 & Can. e Inc. & Mand & Indet & - & - & 33 & 33 \\
\hline F41 143 & Can. e Inc. & Mand & Indet & - & - & 12 y 15 & $12-15$ \\
\hline F41 386 & Can. e Inc. & Mand & $\mathrm{Iz}$ & 80 & $15-21$ & 15 & $15-21$ \\
\hline F41 743 & Caninos & Mand & Indet & - & - & 15 & 15 \\
\hline F41 738 & Mlr & Mand & $\mathrm{Iz}$ & 72,3 & $9-12$ & - & $9-12$ \\
\hline E42 154 & Mlr & Mand & $\mathrm{Iz}$ & 69,1 & $33-48$ & - & $33-48$ \\
\hline E41 144 & Can. e Inc. & Mand & $\mathrm{Iz}$ & - & - & $21-33$ & $21-33$ \\
\hline F41 621 & Caninos & Mand & $\mathrm{Iz}$ & 75 & $9-12$ & $11-15$ & $11-15$ \\
\hline E42 972 & Mlr & Mand & $\mathrm{Iz}$ & 81 & $48-72$ & - & 72 \\
\hline F41 673 & Mlr & Mand & Dr & 81,3 & 15 & - & 15 \\
\hline E42 260 & Mlr & Mand & $\mathrm{Iz}$ & 81 & $33-48$ & - & $33-48$ \\
\hline E42 179 & Mlr & Mand & $\mathrm{Iz}$ & 62 & $2,5-6$ & - & 6 \\
\hline F41 761 & Mlr & Mand & Dr & 76,5 & 21 & - & 21 \\
\hline F41 191 & Mlr & Mand & Dr & 84,3 & $48-72$ & - & $49-60$ \\
\hline F41 620 & Mlr & Mand & Dr & 59,2 & 9 & - & $9-12$ \\
\hline F41 780 & Mlr & Mand & Dr & 73 & 12 & - & $12-15$ \\
\hline F41 462 & Mlr & Mand & $\mathrm{Iz}$ & 79,5 & $15-21$ & - & $\begin{array}{ll}15 & -21\end{array}$ \\
\hline E42 250 & Mlr & Mand & $\mathrm{Iz}$ & 75 & 33 & - & 33 \\
\hline F41 561 & Mlr y Pmlr & Mand & $\mathrm{Iz}$ & - & +-33 & - & 33 \\
\hline F41 581 & Mlr & Mand & Dr & 90 & 33 & - & $33+$ \\
\hline F41 618 & Mlr & Mand & $\mathrm{Iz}$ & 83,5 & $21-33$ & - & $33+$ \\
\hline F41 856 & Mlr & Mand & $\mathrm{Iz}$ & - & $15-21$ & - & $15-21$ \\
\hline E4130 & Mlr 3 & Mand & Dr & - & 48 & - & 48 \\
\hline $\mathrm{E} 42 \mathrm{~s} / \mathrm{T}$ & Incs. 2 & Indet & $\mathrm{Iz}$ & - & - & 15 & 15 \\
\hline $\mathrm{F} 41 \mathrm{~s} / \mathrm{T}$ & Pmlr 4 & Indet & Indet & - & 33 & - & 33 \\
\hline F41 588 & Can. e Inc. & Mx & Indet & 81 & 36 & & 36 \\
\hline F41 590 & Can. e Inc. & Mx & Indet & 85 & 30 & - & $33+$ \\
\hline F41 526 & Can. e Inc. & $\mathrm{Mx}$ & Indet & 82 & 36 & - & 36 \\
\hline F41 24 & Can. e Inc. & $\mathrm{Mx}$ & Indet & 79,6 & 36 & - & 36 \\
\hline F41 589 & Can. e Inc. & $\mathrm{Mx}$ & Indet & 81 & 28 & - & $28+$ \\
\hline F41 585 & Mlr & $\mathrm{Mx}$ & Indet & 70 & 15 & - & 15 \\
\hline F41 523 & Mlr & $\mathrm{Mx}$ & $\mathrm{Iz}$ & 74,9 & 15 & - & $15-21$ \\
\hline F41 211 & Mlr & $\mathrm{Mx}$ & Dr & 67 & 15 & - & 15 \\
\hline F41 533 & $\mathrm{Mlr}$ & $\mathrm{Mx}$ & $\mathrm{Iz}$ & 77 & 26 & - & 26 \\
\hline F41 572 & Can. e Inc. & $\mathrm{Mx}$ & $\mathrm{Iz}$ & 76,5 & 26 & - & 26 \\
\hline F41 667 & Mlr & $\mathrm{Mx}$ & Dr & 69 & 26 & - & $12-15$ \\
\hline F41 197 & Mlr & $\mathrm{Mx}$ & $\mathrm{Iz}$ & 69 & 26 & - & $12-15$ \\
\hline F41 442 & Mlr & $\mathrm{Mx}$ & Indet & 63 & 18 & - & $12-18$ \\
\hline
\end{tabular}

Tabla 7. Estimación de edad, según medidas tomadas en mandíbulas, maxilares y elementos dentarios aislados.

\section{Discusión y consideraciones finales}

El conjunto óseo de la tercera ocupación de LV1 presenta una excelente preservación, donde no se observa correlación entre la DMO y el \%MAU de esta colección (ver Santiago 2010). Resulta entonces interesante, en referencia a la edad de los individuos presentes en la muestra, la ausencia de crías recién nacidas y de neonatos, pudiendo interpretarse que la ausencia de esta categoría de edad no es producto de una pérdida atricional de huesos si no 
de la selectividad humana. Es decir, que se predaba sobre grupos de animales recientemente expulsados de las manadas familiares, cuando estos animales se reúnen bien en las manadas mixtas (conformadas en momentos de migración, generalmente en invierno) o bien en las manadas de machos.

Teniendo en cuenta las edades de muerte registradas para LV1 y comparándolas con perfiles de muerte atricionales y catastróficos teóricos (Raedecke 1978, Stiner 1990), para el caso de LV1 se postula que la mayoría de los animales cazados no eran ni muy jóvenes ni muy viejos: la mayor parte se corresponde con ejemplares sub-adultos y adultos jóvenes (Figura 2). El conjunto indica que no han predado sobre los machos y hembras reproductivas, sino que se cazaron animales suficientemente grandes por su rentabilidad (cuando ya han alcanzado el $85 \%$ del peso total de un adulto, Raedecke 1978) e inmediatamente después de ser expulsados de las manadas familiares.

Las edades identificadas informan también de la posible estacionalidad en el uso del sitio. Considerando que en Tierra del Fuego el guanaco tiene sus pariciones anuales limitadas al verano (diciembre / febrero), y que el $85 \%$ de los nacimientos ocurren antes del 30 de enero (Raedecke 1978), la ausencia de crías recién nacidas indicaría que las cacerías de guanacos se realizaron en este lugar entre fines del otoño y fines de primavera. Si bien es posible obtener evidencia de una ocupación estacional concreta, resulta más difícil probar que un sitio no estuvo ocupado o que fue visitado durante otras épocas del año (Kaufmann 2004).

En cuanto a la composición sexual en LV1, los datos arrojan resultados distintos: siguiendo las medidas tomadas en las pelvis se identificaron 4 machos $(80 \%)$ y 1 hembra (20\%). En tanto que siguiendo las medidas tomadas en los caninos y caniniformes se determinaron según los caninos de las mandíbulas 4 machos (esta es la metodología mas precisa) y según los caninos y caniniformes de los maxilares 3 hembras (60\%) y 2 machos (40\%) (como se dijo, esto tiene menor precisión en la determinación, ya que hay superposición entre machos y hembras, en las variables hasta los tres años, según Kaufmann 2008).

A partir de estos datos y teniendo en cuenta la etología del guanaco planteada por diversos autores (Raedecke 1978, Merino y Cajal 1993), es posible inferir el tipo de manadas que los cazadores-recolectores estaban eligiendo para cazar en LV1. Si naturalmente las poblaciones de guanacos se pueden diferenciar en grupos familiares, grupos de machos, machos solitarios, grupos de hembras y grupos mixtos (Raedeke 1978, Kaufmann 2008), entonces tomando todos los datos analizados podríamos afirmar que se estaba predando sobre manadas familiares. Pero si solo nos ceñimos a los datos más precisos, es decir, usando las medidas en caninos mandibulares y pelvis, el panorama se muestra diferente, ya que a través de ambos métodos se demuestra una predominancia de machos en el conjunto analizado.

En Tierra del Fuego, Merino y Cajal (1993) a partir de diversos censos realizados en los meses de enero-febrero de 1984-1989 en un ambiente boscoso de Península Mitre, informan que el $8 \%$ de los machos adultos formaban parte de los grupos familiares, el $79 \%$ de los grupos de machos y el 13\% eran machos solitarios. Estas relaciones podrían variar en otros ambientes como la estepa, pero teniendo en cuenta la proporción de machos y hembras registrados en LV1 y las diversas proporciones en que se encuentran los géneros en los distintos grupos sociales de guanacos, se pueden plantear algunas opciones acerca de los grupos sociales sobre los cuales predaban: 
a) sobre manadas mixtas.

b) sobre manadas de machos.

c) secuencialmente sobre machos solitarios.

Entonces, de acuerdo al conjunto de datos considerados y a cómo se crucen las variables utilizadas, podría optarse por más de una opción. Por el momento, si la tercera ocupación de LV1 se interpreta como un único evento de caza, la opción c, en la cual se esperaría que el registro sea sólo de machos, puede descartarse.

Por lo tanto las opciones a y b son las más plausibles. Si se consideraran solamente los datos obtenidos en las medidas de pelvis y de caninos de las mandíbulas, nos deberíamos inclinar por la opción b. Pero si utilizamos los datos obtenidos en las piezas dentales de los maxilares la opción a es la más adecuada, ya que los porcentajes de hembras son similares.

Por el momento descartamos que se esté predando sobre manadas familiares porque al cruzar los datos con el perfil etario estamos en presencia de animales en edad de ser expulsados de este tipo de manadas, ya que según Raedecke es entre los 6 y 12 meses que los machos dominantes expulsan a sus crías machos de las manadas familiares $(1978: 110,111)$ tolerando más tiempo a las crías hembras.

Predar sobre los machos de guanaco tiene algunas ventajas; los mismos tienen una mejor condición física a lo largo de todo el año, ya que están exentos de los gastos energéticos extra a los cuales están sometidas las hembras con la gestación y el parto (Raedecke 1978). Cazando machos también se incurre (consciente o incoscientemente) en una preservación del recurso, pues se resguardan las hembras reproductivas, que proveerán de más individuos en las temporadas siguientes. Hay que tener en cuenta que en las manadas de machos es posible encontrar algunas hembras jóvenes, que pueden haber sido expulsadas de su manada por el macho predominante; en algunos de los grupos de machos se ha identificado hasta un 15\% de hembras (Saba y Lamo 1990).

En cuanto a la ausencia de chulengos en la muestra, si como dijimos, la preservación diferencial no es la causa de ello, la interpretación entonces es que al procurar no capturar las crías también se resguarda el recurso como un todo, ya que se salva uno de los eslabones más débiles de la población de guanacos, y se preserva el mismo a futuro. Al mismo tiempo, esta ausencia reflejaría -tal como se mencionó antes- una cierta estacionalidad en el uso de este espacio, siendo visitado por lo menos desde el otoño a fines de la primavera.

En LV1, los cazadores de la 3ra ocupación han optado por la captura preferentemente de machos jóvenes, aunque ocasionalmente hubieran obtenido alguna hembra joven (esta proporcion de animales ha sido observada en las manadas de machos). La interpertación podrá confirmarse o modificarse a la luz de los materiales obtenidos en la campaña más reciente, aún sin analizar completamente.

\section{Agradecimientos}

Una versión preliminar de este trabajo fue presentado en el II Congreso Nacional de Zooarqueología Argentina (Olavarría, mayo 2011). El proyecto PIP 0422-10 financió parcialmente este trabajo. La empresa Roch y Ea María Behety proporcionaron la autorización para el trabajo en el sitio Las Vueltas 1; los fechados se realizaron en University of Arizona AMS Facilities. Julieta Sartori, Melina Coll, Fernanda Bruzzoni y Jimena Oría participaron en los trabajos de campo de 2008. Cristian Kaufmann 
y un evaluador anónimo, a través de sus comentarios, contribuyeron a mejorar el manuscrito. Los autores son los únicos responsables de las ideas aquí expuestas.

Nota

${ }^{1}$ Nuevos registros de campo de la excavación 2012, aún sin analizar en detalle-solo vistos al momento de la extracción-, nos llevarán seguramente a replantear estas hipótesis ante el incremento en el registro de elementos dentarios y pélvicos.

\section{Bibliogrfía citada}

Kaufmann, C.A.

2004 La fusión ósea como indicador de edad y estacionalidad en guanaco (Lama guanicoe). Contra viento y marea: Arqueología de Patagonia (ed. por M.T.Civalero, P. Fernandez y G. Guraieb), pp. 477-487. Instituto Nacional de Antropología y Pensamiento Latinoamericano y Sociedad Argentina de Antropología. Buenos Aires.

2008 Metodologías para la construcción de perfiles de mortalidad y determinación de la estacionalidad en restos óseos de Guanaco (Lama guanicoe): su aplicación a sitios arqueológicos de la región pampeana. Tesis doctoral Inédita, Facultad de Ciencias Naturales y Museo, UNLP, La Plata.

2009 Estructura de edad y sexo en guanaco. Estudios actualísticos y arqueológicos en Pampa y Patagonia. Sociedad Argentina de Antropología. Buenos Aires.

Kaufmann, C. y G.L. L'Heureux

2009 El dimorfismo sexual en guanacos (Lama guanicoe). Una evaluación osteométrica de elementos poscraneales. Revista del Museo de Antropología. Dossier - Arqueología 2: 181-198.

Marean, C.W.

1997 Hunter-Gatherer Foraging Strategies in Tropical Grasslands: Model Building and Testing in the East African Middle and Later Stone Age. Journal of Anthropological Archaeology 16: 189-225.

Merino, M. y J. Cajal

1993 Estructura social de la población de guanacos (Lama guanicoe Muller, 1776) en la costa norte de Península Mitre, Tierra del Fuego, Argentina. Studies on neotropical fauna and environment 28 (3):129-138.

Pagano, M. I. y A. M. Aguerre

2001 Desgaste dentario y determinación de edad en guanacos arqueológicos: una puesta al día. El uso de los camélidos a través del tiempo (ed. por G. Mengoni Goñalons, D. Olivera y H. Yacobaccio), pp. 203-220. Del tridente. Buenos Aires.

Puig, S. y S. Monge

1983 Determinación de la edad en Lama guanicoe (Müller). Deserta 7: 246-270.

Raedecke, K.

1978 El guanaco de Magallanes, Chile. Su distribución y su biología. Santiago de Chile.

Saba, S. y D. A. De Lamo

1990 Donde estan las hembras de guanaco? Patagonia Agropecuaria. 21, 38-40. 
Santiago, F.

2010 La ocupación humana en el norte de Tierra del Fuego durante el Holoceno medio y tardío. Su vinculación con el paisaje. Tesis doctoral Inédita, Facultad de Ciencias Sociales, UNICEN, Olavarría.

Santiago, F. y M. Salemme

2009 Las Vueltas 1: un sitio de matanza de guanacos del Holoceno Tardío en el norte de Tierra del Fuego (Argentina). Arqueología de Patagonia. Una mirada desde el último confín (ed. por M. Salemme, F. Santiago, M. Alvarez, E.L. Piana, M. Vázquez y M.E. Mansur), pp. 785804. Editorial Utopías. Ushuaia.

Santiago, F. y M. Salemme 2010 A guanaco kill site in Tierra del Fuego, Argentina. Before Farming 2/2010: 1-17.

Santiago, F., G. Bujalesky y M. Salemme

2007 Prospección arqueológica en la cuenca del río Chico. Tierra del Fuego, Argentina. Arqueología de Fuego-Patagonia. Levantando piedras, desenterrando huesos... y develando arcanos (ed. por F. Morello, A. Martinic, A. Prieto y G. Bahamonde), pp. 357-363. Ediciones CEQUA. Punta Arenas (Chile).

Sarasqueta, D.V.

2001 Cría y Reproducción de Guanacos en Cautividad Lama guanicoe. INTA EEA Bariloche, Fauna Silvestre. Bariloche.

Stiner, M.C.

1990 The use of mortality patterns in archaeological studies of hominid predatory adaptations. Journal of Anthropological Archaeology 9: 305-351.

Weaver, T.D., Boyko, R.H., Steele, T.E., 2011. Cross-platform program for likelihood-based statistical comparisons of mortality profiles on a triangular graph, Journal of Archaeological Science 38: 2420-2423. 